\section{Too quiet to hear a whisper}

\section{P. Jung and K. Wiesenfeld}

Add some noise, and many dynamic systems respond to weak signals more strongly. Some sort of threshold process was thought to be necessary for this effect, but a new model removes the restriction.

ए luctuations usually destroy order, yet there are situations where noise plays a surprisingly constructive role. This realization has triggered a lot of excitement in recent years, most notably in the study of the phenomenon of stochastic resonance, which is observed in experiments including ring lasers, superconducting magnetometers and sensory neurons. Now it seems that stochastic resonance is even more common than previously suspected, according to a theory by Sergey Bezrukov and Igor Vodyanoy reported on page 319 of this issue ${ }^{1}$, with possible implications for systems ranging from mesoscopic electronic devices to signal transduction in ion channels through cell membranes.

The basics of stochastic resonance were laid out in the early 1980 s, with speculation that noise might be responsible for the apparent periodicity of ice ages (the Milankovitch cycles) by amplifying tiny periodic variations in the Earth's orbit ${ }^{2,3}$. There followed a wealth of theoretical and experimental work, leading to a well-settled understanding of the effect. The conditions that lead to stochastic resonance are fairly simple and robust, which is why it has been observed so readily in so many different experiments.

Over time, the original notion of stochastic resonance has widened to include a number of different mechanisms. The unifying feature is that noise can improve a system's sensitivity to weak signals, such as periodic inputs. One measure of sensitivity is the out-

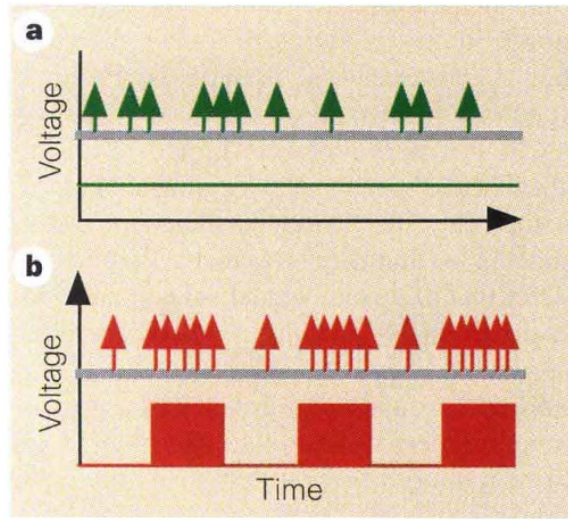

Figure la, A typical spike train, generated with constant input, compared with (b) a spike train generated by a periodic rectangular input. The properties of the input signal are reflected in the spike pattern. put signal-to-noise ratio, and a signature of stochastic resonance is when this ratio passes through a maximum as a function of the applied noise intensity. The earliest research focused on bistable systems (a class that includes the ice-age model), but has since been expanded to include excitable systems ${ }^{4}$ that have a single rest-state. Study of these systems was motivated by the potential relevance of stochastic resonance to neuronal dynamics.

Simple cartoon versions of excitable systems known as threshold detectors ${ }^{5}$ have been considered intensively because they are accessible to full analytical treatment. In these models, the detector responds (bysending out a pulse, for example) whenever the sum of the signal and the noise crosses a threshold. A crucial ingredient for a system to show stochastic resonance was thought to be the existence of a potential barrier, or a threshold of some sort. Bezrukov and Vodyanoy, however, say otherwise.

They consider a theoretical device which spits out a random spike train with a constant spike rate. In their model, this rate depends exponentially on an external input, such as a voltage. So as the input varies in time, the spike rate changes: the spiking pattern thus encodes, by its inhomogeneity, the input signal (Fig. 1). The input is the sum of a weak sinusoidal signal and external noise. A key aspect of the model is that - in contrast to earlier work on spike trains generated by excitable detectors - there is no threshold

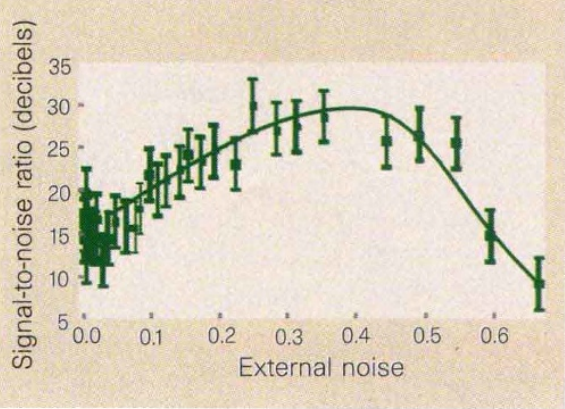

Figure 2 A strongly reverse-biased p-n silicon diode is driven by a weak sinusoidal voltage. With no external noise, the output signal-tonoise ratio is very small. Adding gaussian noise to the weak input, the signal-to-noise ratio increases to a maximum at a finite noise intensity. or potential barrier. That is, an arbitrarily weak signal affects the spike train even in the absence of external noise.

Bezrukov and Vodyanoy calculate the signal-to-noise ratio of the outgoing spike train. The main result is that the ratio goes through a maximum as the external noise strength is increased, a signature of stochastic resonance. In order to carry through the analysis fully, they make some assumptions: that there are no spike-spike correlations; that the amplitude of the sinusoidal signal is small; that the spike rate is exponentially dependent on the inputs; and that the averaging over the inherent randomness and external noise can be performed separately (that is, there is no causal relationship).

In real systems these points (especially the last one) will need investigation, but the model is certainly plausible. Indeed, Fig. 2 shows data supporting it for one of the candidate systems listed by Bezrukov and Vodyanoy (B. Meadows and W. Ditto, personal communication). The output signalto-noise ratio for a strongly reverse-biased electronic diode is plotted against input noise intensity. In a diode, the output current consists of clusters of charge carriers with inherent randomness, and this output can be viewed as a spike train. The current is the mean flow rate of charge carriers, and depends exponentially on the applied voltage. Although it remains to be explored how far the model assumptions are fulfilled for this particular system, the data clearly show the stochastic resonance peak.

By doing away with a sharp threshold, Bezrukov and Vodyanoy show that stochastic resonance applies to a new class of systems. The new requirement is that the spike rate depends exponentially on the input, but that is a fairly common property. For example, the rate at which electrons boil off a conductor (thermionic emission) depends exponentially on the surface potential, and the current through ion channels is similarly sensitive to a voltage difference across the cell membrane. In fact, an earlier experiment ${ }^{6}$ by Bezrukov and Vodyanoy on alamethicin ion channels in a lipid bilayer was a motivating factor for this newer theoretical work.

It is intriguing to speculate to what extent this property has a function: is stochastic resonance widely exploited in biological systems to detect weak signals? This remains very much an open question, but the step taken by Bezrukov and Vodyanoy is a further temptation to explore the role of noise in signal transduction.

P. Jung and K. Wiesenfeld are in the School of

Physics, Georgia Institute of Technology, Atlanta,

Georgia 30332-0430, USA.

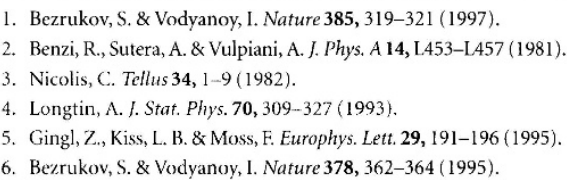

